\title{
Enhanced Bandwidth Utilization in Image Steganography with Enhanced Data Security
}

\author{
Balkrishan \\ Department of CE, Yadavindra College of \\ Engineering, Punjabi University, Guru Kashi \\ Campus, Talwandi Sabo-151302 \\ District: Bathinda Punjab, India
}

\author{
Amar Partap Singh \\ Department of ECE, Sant Longowal Institute of \\ Engineering and Technology,Longowal-148106 \\ District: Sangrur, Punjab, India
}

\begin{abstract}
In this paper, a new method is proposed for image steganography that involves double encryption as well as compression of a message followed by its subsequent hiding in a digital image. In the first instance, original data is encrypted using flexible matrix. Further, encrypted data is encrypted and compressed using Chinese Remainder Theorem (CRT) for extra layer of security and increased the data hiding capacity. The proposed method enhances bandwidth utilization besides ensuring three layered security to the message. The underlying principle of this method involves decomposition of each image-pixel into two blocks. One block is called Parity Reflecting Block (PRB) whereas other is known as Pixel Adjustment Block (PAB). The information about hidden bit is reflected by parity condition in the Parity Reflecting Block. The Pixel Adjustment Block is used to perform local pixel adjustment in order to reduce the degradation effect in the cover image produced as a result of alteration in the moderate bit. The performance of the proposed algorithm is evaluated in terms of Image Quality Measures (IQM) including Mean Square Error (MSE), PeakSignal-to-Noise Ratio (PSNR), Entropy, Correlation, Mean Value and Standard Deviation. Security analysis is also carried by comparing the histograms of the cover and stegoimages. The results of this study are quite promising.
\end{abstract}

\section{General Terms}

Information Security

\section{Keywords}

Data Hiding, Chinese Remainder Theorem, CryptoCompressed-Data, Flexible Matrix, Parity

\section{INTRODUCTION}

Data encryption [1-4] \& compression [2-4] are pivotal for proper storage and transmission of data. The rapid growth in the internet coupled with high bandwidth requirement has propelled the explosive growth of information communication. This type of advancement in the field of data communication has hiked the fear of getting the data snooped at the time of sending it from sender to the receiver. So, information security has already become an important part of data communication. In order to address this issue of information security, data-encryption as well as its hiding [516] plays an important role. Data hiding conceals the existence of secret message while cryptography protects its contents. The word steganography is derived from the Greek words- stegos meaning roof or cover and graphia meaning writing, i.e., it is an art of hiding information in such a way that communication takes place without any failure [5-7]. The objective of modern steganography is to keep the payload (embedded information) undetected, but the steganographic systems, because of their invasive nature, may leave behind somewhat traces in the cover image [8]. Steganography techniques use different carriers (cover medium in digital format) to hide data. These carriers may be network packets, floppy disk, hard drive, amateur radio waves [9] or general computer files such as text, image, audio, video, etc. [5, 9-12]. Data hiding capacity \& invisibility are the two important parameters used to evaluate the effectiveness of the data hiding technique [14]. In [5] crypto data was embedded into the moderate significant bit of pixel of the cover image by parity condition of bits. The weakness of this method is that author used only lower bits for pixel adjustment and not used the upper bits for re-adjustment of the pixels of the stego image.

The simultaneous data encryption and compression technique is an attempt to provide a solution for optimal bandwidth utilization, space required for data storage and the encryption problems at the same time [2]. Number theory based data encryption and compression is an algorithm that employs Chinese Remainder Theorem (CRT) in order to generate and solve congruencies. However, several methods are available for data or image compression including JPEG-LS, SPIHT, JPEG2000, CALIC, etc. These are standards that use some kind of transform including Discrete Fourier Transformation (DFT), Discrete Cosine Transformation (DCT) \& Discrete Wavelet Transformation (DWT) [3]. There are two main drawbacks of traditional transformation based compression methods: (a) In transform techniques, data is transformed from one domain to another, (b) Moreover, these techniques do not encrypt and compress image and/or data in single step [4]. However, such problems of traditional methods of compression are addressed in the proposed approach in which only basic mathematical operations are employed instead of transformation of the data. It also ensures encryption and compression of data in a single step.

Therefore, in the present work, a new type of image steganography is described that combines three operationscryptography, data compression and steganography in a simultaneous manner. In the first instance, the message is encrypted using flexible matrix [15] serving the purpose of a new type of symmetric key. The data byte is assigned a row number and column number from the flexible matrix itself. All row and column numbers are combined to form one dimensional (1-D) array. This process implements first layer of security to the data. It provides 256 combinations for data byte instead of single combination (remainder and quotients) when data byte is divided by 16 in the CRT. After this, enciphered data is again encrypted as well as compressed using Chinese Remainder Theorem (CRT). In addition to this, it also provides an additional layer of security to the original message and also increases the data hiding capacity of the 
cover image by compressing the secret data. At the end, crypto-compressed-data is embedded into the pixel of cover image using moderate-bit substitution thus providing third layer of security in the form of camouflage. Moderate bit substitution is achieved in such a way that no appreciable distortion is observed in the cover image. To improve the image quality further, a pixel adjustment process is also applied at each pixel of the cover image where alteration has occurred due to moderate significant bit substitution. This is done by slight adjustment of other bits in the image pixel without any damage to the secret data. To evaluate the visual quality of stego-image, Image Quality Measures (IQM) are evaluated including Mean Square Error (MSE), Peak-Signalto-Noise Ratio (PSNR), Entropy, Correlation, Mean Value and Standard Deviation [17]. The proposed method is applied on different standard test-images of size $256 \times 256$ serving the purpose of good cover. Extensive experimental results proved that the hidden data remains invisible and there is no appreciable visual distortion in the image at all.

\section{CHINESE REMAINDER THEOREM}

The Chinese Remainder Theorem (CRT) is a good application of number theory to other fields. It is based on the algorithm of linear and modular congruencies. Congruence is nothing more than a statement about divisibility [4]. The Chinese Remainder Theorem is mainly based on the system of linear congruencies

$a=b(\bmod n)$ which can be reduced to a set of $a=b\left(\bmod n_{i}\right)$, where $n_{1}, n_{2} \ldots n_{k}$ are prime factors of $\mathrm{n}$.

\section{(a) Theorem}

Let $n_{1}, n_{2} \ldots n_{k}$ denote $\mathrm{k}$ positive integers which are relatively prime numbers, and let $a_{1}, a_{2} \ldots a_{k}$ denote any $\mathrm{k}$ integers. Then the congruencies $X=\bmod \left(a_{i}, n_{i}\right)$, where $\mathrm{i}=1,2, \ldots, \mathrm{k}$ have common solutions. Any two solutions are congruent Modulo $n_{1}, n_{2} \ldots n_{k}$

$Y=X(\bmod P)$ Where $P=n_{1} * n_{2} * \ldots n_{k}$

$X=\left(\left(a_{1} * N_{1} * x_{1}\right)+\cdots+\left(a_{k} * N_{k} * k\right)\right)(\bmod P)$

i.e. $X=\sum\left(a_{i} * N_{i} * x_{i}\right)(\bmod P)$

Where, $N_{k}=\frac{P}{n_{k}}$ in which $x_{k}$ satisfied

$N_{i} * x_{i}=1\left(\bmod n_{i}\right)$

The remainder of the solved congruencies $\mathrm{X}$ is transmitted. At the receiving end, $a_{i}$ are found using $a_{i}=X\left(\bmod n_{i}\right)$ and the original data is reconstructed by row number and column number of flexible matrix.

(b) Numerical Example of data encryption and compression using CRT

Let $n_{1}=17, n_{2}=18, n_{3}=19 \& n_{4}=23$

which are relatively prime

Let $a_{1}=10, a_{2}=14, a_{3}=9 \& a_{4}=8$

Encryption:

$$
\begin{gathered}
P=133722 \\
N_{1}=7866, N_{2}=7429, N_{3}=7038 \& N_{4}=5814 \\
x_{1}=10, x_{2}=7, x_{3}=12 \& x_{4}=9, X=18914
\end{gathered}
$$

Decryption:

$$
\begin{array}{r}
a_{i}=\bmod \left(X, n_{i}\right) \\
a_{1}=10, a_{2}=14, a_{3}=9, a_{4}=8
\end{array}
$$

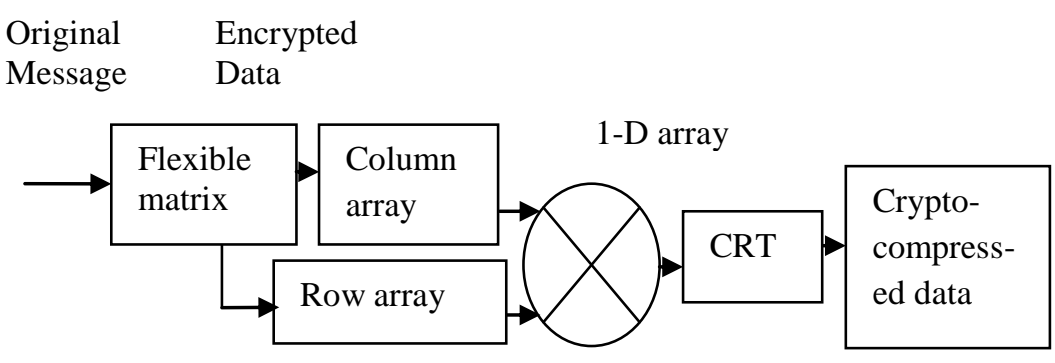

Fig.1 Proposed Method for Data Encryption and Compression

\section{PROPOSED METHOD}

The secret data is first encrypted using flexible matrix proposed by the authors of the present paper in their earlier work [15]. This matrix is reproduced in Table-1 with new entries for the sake of illustration [15]. The enciphered data is further re-encrypted \& then compressed using CRT. The complete process is illustrated in Fig.1. The number theory based technique is applicable for encryption application by suitable selection of relatively prime numbers $\left(\mathrm{n}_{\mathrm{i}}\right)$. Data compression depends on the block size used in CRT.

Relative prime numbers are chosen in such a way that these are larger than any value in one dimensional (1-D) array. During decoding, the same combination of $n_{i}$, that are selected for encoding should be applied correctly. To achieve safe transfer of data and obtain better results of embedding, moderate-bit substitution method is proposed in the present work for hiding crypto-compressed-data in a digital image. In this paper, for compression purpose, the block size $(1 \times \mathrm{K})$ with $\mathrm{K}=4$ is taken. Depending on the amount of compression requirement, a large block size can also be considered.

\subsection{Data Encryption and Compression using CRT}

Consider a data of $\mathrm{n}$ characters and assign the row number and column number of $\mathrm{n}$ characters using flexible matrix [15]. The Row number and column number are combined to form 1- D array. Pad the 1-D array with zeros to make a block size of 10 or 8 or 4 . Now, 1-D array is solved using the Chinese Remainder Theorem for block of 4 . In equation (2) Ni and xi are pre-calculated as coefficients and these values need not be calculated for every X. All ai $(i=1, \ldots, m$, where $m$ is the elements in 1-D array) are the 1-D array values after encryption using flexible matrix. The reason for using Chinese Remainder Theorem for solving the linear congruencies is to reduce a bigger number to a smaller representation. For data of size $1 \times 3000$ bytes and block size 4 , all 1500 or less $X$ are computed. After computing all $X$, the frequency of each distinct $\mathrm{X}$ and their counts are determined. All X are sorted in descending order of their count. A table of unique $\mathrm{X}$ and an equivalent smaller code is generated. Using this table, each $\mathrm{X}$ obtained is encoded into this smaller code. Data compression ratio was 1.35 for 3000 bytes. For the sake of clarification, the complete process is illustrated here.

\subsection{Data Decryption and Decompression}

Data decoding is performed at the receiving end. At the receiver, Ai is found for each X using the equation (4). Single 1-D array of all Ai is divided into two equal size arrays (Row number \& Column number).The original pixel values are then reconstructed using the flexible matrix. 


\subsection{Illustration}

\begin{tabular}{|c|c|}
\hline $\begin{array}{l}\text { Original message to be } \\
\text { embedded }\end{array}$ & Image steganography \\
\hline $\begin{array}{l}\text { Data byte/ASCII value of } \\
\text { character }\end{array}$ & $\begin{array}{rrrlrr}105 & 109 & 97 & 103 & 101 & 32 \\
115 & 116 & 101 & 103 & 97 & 110 \\
111 & 103 & 114 & 97 & 112 & 104 \\
121 & & & & & \\
\end{array}$ \\
\hline $\begin{array}{l}\text { Encrypt the message using } \\
\text { flexible matrix }\end{array}$ & 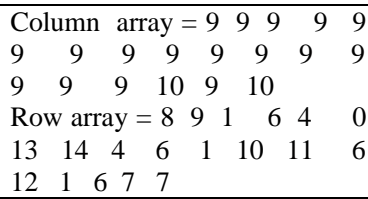 \\
\hline $\begin{array}{l}\text { Combine the column and } \\
\text { row array to get a } 1-\mathrm{D} \text { array } \\
\text { and pad with zeros such that } \\
\text { total values in 1-D arrays } \\
\text { divided by } 4\end{array}$ & $\begin{array}{lccccccccc}\text { 1-D array }= & 9 & 9 & 9 & 9 & 9 & 9 \\
9 & 9 & 9 & 9 & 9 & 9 & 9 & 9 \\
9 & 9 & 10 & 9 & 10 & 8 & 9 & 1 & 6 \\
4 & 0 & 13 & 14 & 4 & 6 & 1 & 10 \\
11 & 6 & 12 & 1 & 6 & 7 & 7 & 0 & 0\end{array}$ \\
\hline $\begin{array}{lrr}\text { Further } & \text { encrypt } & \text { and } \\
\text { compress } & \text { crypto data using } \\
\text { Chinese } & \text { Remainder } \\
\text { Theorem } & \end{array}$ & $\begin{array}{l}\text { Calculate the values of } X \text { and } \\
\text { assign the smaller code to each } \\
X\end{array}$ \\
\hline $\begin{array}{l}\text { Data embedding into cover } \\
\text { image to obtain stego-image }\end{array}$ & $\begin{array}{l}\text { Data embedding at Moderate } \\
\text { Significant Bit position }\end{array}$ \\
\hline
\end{tabular}

\subsection{Crypto-compressed-data hiding}

To understand the process of crypto-compressed-data hiding, it is assumed that a cover image is composed of pixels with odd or even number of one's. In the pixel of cover image, bit is altered at moderate position depending upon the parity condition of the bits counted from moderate position to the most significant bit (MSB). All the lower bits from moderate position to Least Significant Bit (LSB) are used for local pixel adjustment. The procedure used for moderate bit alteration followed by local pixel adjustment is narrated below:

I. If the PRB is of odd parity and message bit is 1 , then there is no change in both the pixel blocks of the image pixel. The odd parity condition in the PRB reflects that the stored bit is 1 [16].

II. If the PRB is of odd parity and the message bit is 0 , then complement the $4^{\text {th }}$ moderate significant bit. Convert all the lower bits to 1 if moderate bit is changed to 0 or vice-versa otherwise [16].

III. If the PRB is of even parity and the message bit is also 0 , then there is no change in both the pixel blocks of the image pixel. The even parity condition of the PRB reflects that image pixel stores 0 as the message bit [16].

IV. If the PRB is of even parity and the secret message bit is 1 , then complement the $4^{\text {th }}$ moderate significant bit. Convert all the lower bits to 1 if moderate bit is changed to 0 or viceversa otherwise [16].

\subsection{Pixel Adjustment Process}

Following are the two steps used in pixel adjustment process.

\section{Pixel Adjustment [16]}

If crypto-bit to be embedded is equal to the 4th LSB, then there is no need for doing any pixel adjustment. However, if crypto-bit to be embedded is not equal to 4th LSB, pixel adjustment is performed by using first three LSBs. The underlying logic for the same is detailed below:

(a) If crypto-bit to be embedded is one, then pixel is adjusted by changing first three LSBs to zero.

(b) If crypto-bit to be embedded is zero, then pixel is adjusted by changing first three LSBs to one.

\section{Post pixel adjustment}

In this step, post pixel adjustment is applied further improve the visual quality of stego image obtained after pixel adjustment. This process involves only two bits, i.e., $5^{\text {th }}$ and $6^{\text {th }}$ bits. Let $\mathrm{P}, \mathrm{P}^{\prime}$ and $\mathrm{P}$ " are the values of $i^{\text {th }}$ pixel in the cover-image, modified pixel of stego-image after pixel adjustment and modified pixel of stego image after post pixel adjustment, respectively. Calculate the error (D1) between modified pixel of stego-image after pixel adjustment and original pixel of cover image, i.e., D1 = abs (P-P'). If D1 > 4, then post pixel adjustment is required otherwise there is no need of doing post pixel adjustment. The value of D1 $>4$ is chosen in such a way that it gives better visual quality as compared to its other values including $\mathrm{D}=1,2,3,5,6,7$ or 8 . The underlying logic for the same is detailed below:

(a) If after the process of embedding, $4^{\text {th }} \& 5^{\text {th }}$ LSBs are unequal and $4^{\text {th }} \& 6^{\text {th }}$ LSBs are equal, then post pixel adjustment is performed by complementing $1^{\text {st }}, 2^{\text {nd }}, 3^{\text {rd }}, 5^{\text {th }}$ and $6^{\text {th }}$ bits of $\mathrm{p}^{\prime}$.

In fact, the proposed method provides a three layered security to protect the hidden data. First, cryptography technique is used to protect the information. Secondly, data hiding capacity of cover image is increased by encrypting and compressing the crypto data using CRT. Then, the cipher information so obtained is embedded in the cover image. The data hiding procedure is illustrated in Fig.2. 


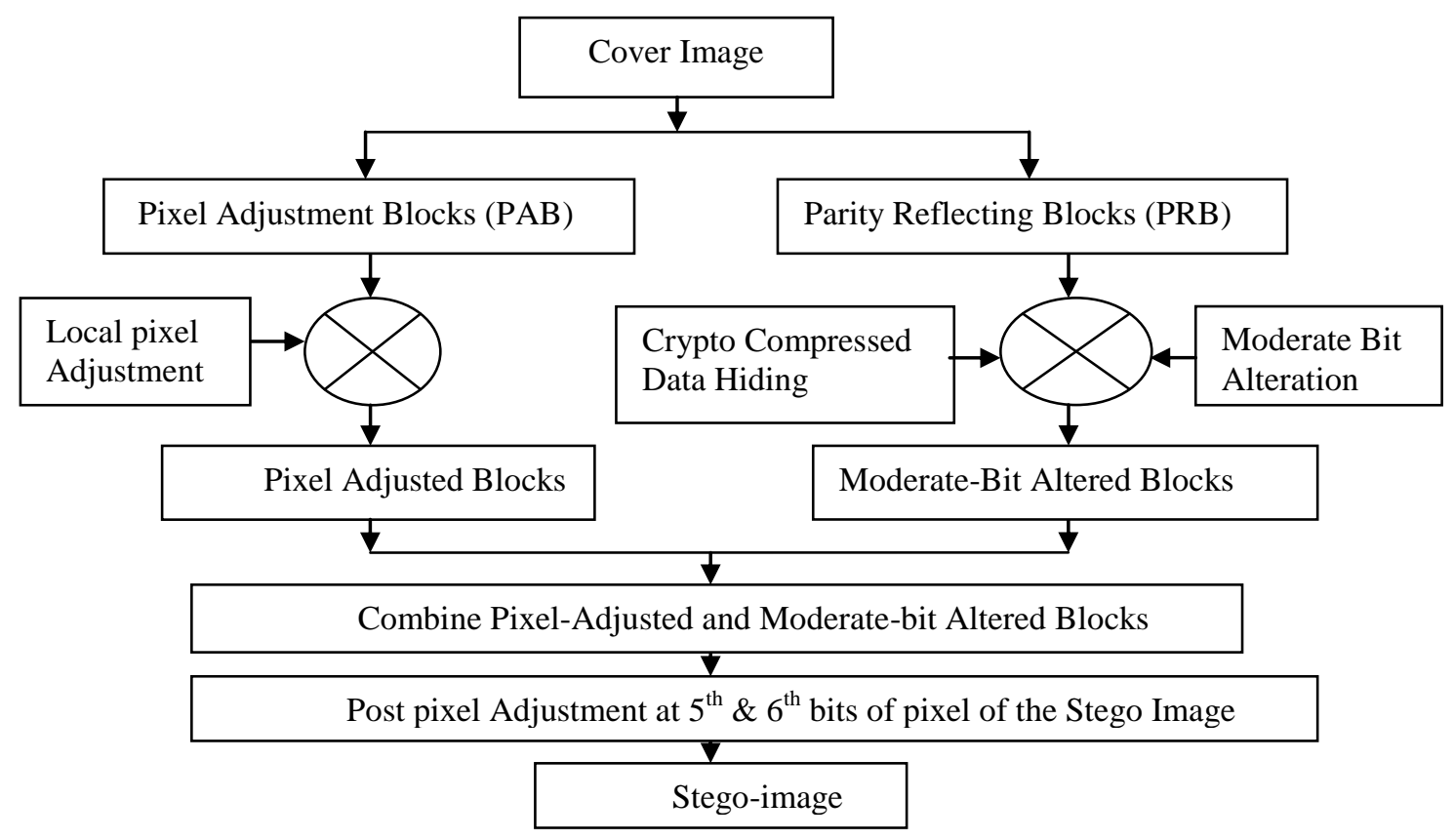

Fig 2: Moderate-Bit Altered Image Steganography

\section{PROPOSED ALGORITHM FOR HIDING CRYPTO COMPRESSED DATA}

\subsection{Message Ciphering \& Embedding}

Step-1: Save secret message as a text file.

Step-2: Commencing with first character, read secret message character-wise from saved text file.

Step-3: Encrypt each character into row and column numbers using flexible matrix.

Step-4: Repeat Step-3 for all characters in the saved text file to obtain a row and column numbers.

Step-5: Combine all the row and column numbers into 1-D array.

Step-6: Pad the 1-D array with zeros such that total values in array must be divided by 4 for a block size of 4 .

Step-7: Further encrypt and compress the ciphered data by applying the CRT using block of 4 values of 1-D array to calculate the value of $X$.

Step-8: Repeat step 7 for all values in 1-D array to obtain the values of $\mathrm{X}$.

Step-9: After computing all $X$, the frequency of each distinct $X$ and their counts are determined. All X are sorted in descending order of their count. A table of unique $\mathrm{X}$ and an equivalent smaller code is generated. Using this table, each X obtained is encoded into this smaller code.

Step-10: All value of $X$ and smaller codes are converted into equivalent binary number (all $\mathrm{X}$ and smaller codes have equal number of bits by padding with zeros from left side) and make a binary string.

Step-11: Read each pixel of the cover image commencing with first pixel.

Step-12: Convert each pixel into equivalent eight-bit binary number called image byte.

Table 1. Proposed 16×16 Matrix for Enciphering Data [15]

\begin{tabular}{|c|c|c|c|c|c|c|c|c|c|c|c|c|c|c|c|c|}
\hline $\begin{array}{c}\text { Column Number } \\
\text { Row Number }\end{array}$ & 0 & 1 & 2 & 3 & 4 & 5 & 6 & 7 & 8 & 9 & 10 & 11 & 12 & 13 & 14 & 15 \\
\hline 0 & 192 & 193 & 194 & 195 & 196 & 197 & 198 & 199 & 200 & 201 & 202 & 203 & 204 & 205 & 206 & 207 \\
\hline 1 & 208 & 209 & 210 & 211 & 212 & 213 & 214 & 215 & 216 & 217 & 218 & 219 & 220 & 221 & 222 & 223 \\
\hline 2 & 224 & 225 & 226 & 227 & 228 & 229 & 230 & 231 & 232 & 233 & 234 & 235 & 236 & 237 & 238 & 239 \\
\hline 3 & 240 & 241 & 242 & 243 & 244 & 245 & 246 & 247 & 248 & 249 & 250 & 251 & 252 & 253 & 254 & 255 \\
\hline 4 & 143 & 145 & 146 & 147 & 148 & 149 & 150 & 151 & 152 & 153 & 154 & 155 & 156 & 157 & 158 & 159 \\
\hline 5 & 160 & 161 & 162 & 163 & 164 & 165 & 166 & 167 & 168 & 169 & 170 & 171 & 172 & 173 & 174 & 175 \\
\hline 6 & 176 & 177 & 178 & 179 & 180 & 181 & 182 & 183 & 184 & 185 & 186 & 187 & 188 & 189 & 190 & 191 \\
\hline 7 & 64 & 55 & 66 & 67 & 68 & 69 & 70 & 73 & 72 & 71 & 74 & 75 & 76 & 77 & 78 & 79 \\
\hline 8 & 80 & 81 & 82 & 87 & 84 & 85 & 86 & 83 & 88 & 89 & 90 & 91 & 92 & 93 & 94 & 95 \\
\hline 9 & 32 & 97 & 99 & 100 & 101 & 102 & 103 & 104 & 105 & 109 & 110 & 111 & 114 & 115 & 116 & 117 \\
\hline 10 & 46 & 98 & 106 & 107 & 108 & 113 & 112 & 121 & 118 & 119 & 120 & 122 & 124 & 125 & 126 & 127 \\
\hline 11 & 128 & 129 & 130 & 131 & 132 & 133 & 134 & 135 & 136 & 137 & 138 & 139 & 140 & 141 & 142 & 144 \\
\hline 12 & 0 & 1 & 2 & 3 & 4 & 5 & 6 & 7 & 8 & 9 & 10 & 11 & 12 & 13 & 14 & 15 \\
\hline 13 & 16 & 17 & 18 & 19 & 20 & 21 & 22 & 23 & 24 & 25 & 26 & 27 & 28 & 29 & 30 & 31 \\
\hline 14 & 123 & 33 & 34 & 35 & 36 & 37 & 38 & 39 & 40 & 41 & 42 & 43 & 44 & 45 & 96 & 47 \\
\hline 15 & 48 & 49 & 50 & 51 & 52 & 53 & 54 & 65 & 56 & 57 & 58 & 59 & 60 & 61 & 62 & 63 \\
\hline
\end{tabular}


Step-13: Convert image byte into two blocks-Pixel Adjustment Block and Parity Reflecting Block.

Step-14: Determine the parity of the Parity Reflecting Block (PRB) \& read first crypto-compressed-bit.

a) If the PRB is of odd parity and message bit is 1 , then there is no change in both the pixel blocks of the image pixel. The odd parity condition in the PRB reflects that the stored bit is 1 .

b) If the PRB is of odd parity and the message bit is 0 , then complement the $4^{\text {th }}$ moderate significant bit. Convert all the lower bits to 1 if moderate bit is changed to 0 or vice-versa otherwise.

c) If the PRB is of even parity and the message bit is also 0 , then there is no change in both the pixel blocks of the image pixel. The even parity condition of the PRB reflects that image pixel stores 0 as the message bit.

d) If the PRB is of even parity and the secret message bit is 1 , then complement the $4^{\text {th }}$ moderate significant bit. Convert all the lower bits to 1 if moderate bit is changed to 0 or viceversa otherwise.

Step-15: Go to next image byte and next crypto-compressedbit and repeat the Steps-12 to 14 until all the cryptocompressed bits of the secret message are embedded into the PRBs of the cover image.

\subsection{Message Extraction \& Decryption}

Step-1: Read the pixel of stego-image starting from first pixel.

Step-2: Convert each pixel value into equivalent binary number called image byte.

Step-3: Extract first crypto-compressed-bit by determining the parity condition of the Parity Reflecting Block. If it is odd parity then embedded crypto-bit is 1 otherwise it is 0 .

Step-4: Repeat steps 2 to 3 until all the crypto-compressedbits of the secret message are extracted.

Step-5: Divide the crypto-compressed-bits for values of $\mathrm{X}$ and smaller codes.

Step-6: Smaller codes are also replaced with $\mathrm{X}$ values.

Step-7: Apply the equation (4) and $A_{i}$ of each $X$ using the equation.

Step-8: Repeat step 7 for all values of X.

Step-9: Combine all $\mathrm{A}_{\mathrm{i}}$ to form a single 1-D array. Divide the 1-D array into two equal sized arrays (Row \& Column). The original pixel values are then reconstructed using the flexible matrix.

Step-10: Save all characters in the form of text file.

\section{RESULTS AND DISCUSSION}

In this section, simulation results of the proposed algorithm are presented by embedding 3000 bytes of crypto-compressed data at moderate significant bit positions of the pixels in the cover image. For experiments, four standard gray scale test images with $256 \times 256$ pixel size are employed as cover images (Lena, Mandrill, Pepper and Cameraman). MATLAB software is used to implement the algorithm and validate the results. Original cover images are shown in Fig. 3(a) along with their corresponding stego-images are shown in Fig. 3 (b) $\&$ (c) that are generated as a result of proposed algorithm. The proposed technique embeds crypto-compressed-data into moderate-significant-bit positions in the image-pixels without causing any appreciable distortion in the cover image. Visual quality of a stego image is an important parameter in evaluating the performance of the proposed algorithm. It is expressed in terms of Image Quality Measure (IQM) parameters including Mean Square Error (MSE), PeakSignal-to-Noise Ratio (PSNR), Entropy, Correlation, Mean Value and Standard Deviation. The performance of the proposed algorithm is summarized in terms of IQM parameters. These results are tabulated in Table-2, Table-3 and Table-4. The results in these tables indicate that PSNR, Entropy, correlation, Mean value and Standard Deviation are decreasing whereas the MSE is increasing as the hidden message bit moves from LSB towards moderate significant bit position. The performance of the proposed method is better than [16] in terms of IQM parameters shown in Tables 2, 3 and 4 .

The histograms of cover and stego-images give clear idea of the security of the transmitted data, i.e., if change in the cover is minimal, then stego system is considered secure. In this context, the effectiveness of the proposed algorithm is also determined in terms of having a comparison of the histograms of cover and stego images as show in Fig.4. The histogram analysis of the stego-images proves that these images look exactly similar to that of the original image. Further, this process provides two additional layers of security to the hidden message. First, cryptography technique is used to protect the information and it requires less computational overhead as compared to other steganographic techniques which transforms cover images into frequency domain. Secondly data hiding capacity of cover image is increased by encrypting and compressing the ciphered data using CRT. It also provides 256 combinations for data byte instead of single combination (remainder and quotients) when data byte is divided by 16 in CRT.

Table 2. Mean Square Error and Peak Signal-to-Noise Ratio resulted from hiding $\mathbf{3 0 0 0}$ bytes of Crypto-Compressed data

\begin{tabular}{|c|l|l|l|l|}
\hline $\begin{array}{l}\text { Test } \\
\text { Image } \\
(256 \times 25 \\
6)\end{array}$ & \multicolumn{2}{l}{$\begin{array}{l}\text { Peak Signal-to-Noise } \\
\text { Ratio (PSNR) }\end{array}$} & \multicolumn{2}{l|}{$\begin{array}{l}\text { Mean Square Error } \\
\text { (MSE) }\end{array}$} \\
\cline { 2 - 5 } & With [16] & $\begin{array}{l}\text { Proposed } \\
\text { Method }\end{array}$ & $\begin{array}{l}\text { With } \\
{[16]}\end{array}$ & $\begin{array}{l}\text { Proposed } \\
\text { Method }\end{array}$ \\
\hline Lena & 42.7959 & 43.6624 & 3.4158 & 2.7980 \\
\hline Goldhill & 42.7764 & 43.3040 & 3.4312 & 3.0387 \\
\hline Mandrill & 42.5498 & 43.2155 & 3.6150 & 3.1012 \\
\hline Camera & 41.7003 & 42.2161 & 4.3959 & 3.9036 \\
\hline
\end{tabular}

Table 3. Entropy and Correlation resulted from hiding $\mathbf{3 0 0 0}$ bytes of Crypto-Compressed data

\begin{tabular}{|c|c|c|c|c|}
\hline \multirow{2}{*}{$\begin{array}{l}\text { Test Image } \\
(256 \times 256)\end{array}$} & \multicolumn{2}{|c|}{ Entropy } & \multicolumn{2}{|c|}{ Correlation } \\
\hline & $\begin{array}{l}\text { With } \\
{[16]}\end{array}$ & $\begin{array}{l}\text { Proposed } \\
\text { Method }\end{array}$ & $\begin{array}{l}\text { With } \\
{[16]}\end{array}$ & $\begin{array}{l}\text { Proposed } \\
\text { Method }\end{array}$ \\
\hline Lena & 5.1151 & 5.1239 & 0.9993 & 0.9994 \\
\hline Goldhill & 5.1016 & 5.1090 & 0.9993 & 0.9994 \\
\hline Mandrill & 5.1695 & 5.1748 & 0.9992 & 0.9993 \\
\hline Camera & 4.8165 & 4.8258 & 0.9995 & 0.9995 \\
\hline
\end{tabular}


Table 4. Mean Value and Standard Deviation resulted from hiding 3000 bytes of Crypto-Compressed data

\begin{tabular}{|c|c|c|c|c|c|c|}
\hline \multirow{2}{*}{$\begin{array}{c}\text { Test Image } \\
(256 \times 256)\end{array}$} & \multicolumn{3}{|c|}{ Mean Value } & \multicolumn{3}{c|}{ Standard Deviation } \\
\cline { 2 - 7 } & Cover image & $\begin{array}{c}\text { With } \\
{[16]}\end{array}$ & $\begin{array}{c}\text { Proposed } \\
\text { Method }\end{array}$ & $\begin{array}{c}\text { Cover } \\
\text { image }\end{array}$ & $\begin{array}{c}\text { With [16] } \\
\text { Method }\end{array}$ \\
\hline Lena & 124.0923 & 124.1127 & 124.0150 & 47.8448 & 47.9462 & 47.9539 \\
\hline Goldhill & 112.0349 & 112.2391 & 112.2100 & 48.5952 & 48.6440 & 48.5932 \\
\hline Mandrill & 102.7616 & 102.7898 & 102.7715 & 48.7939 & 48.7962 & 48.7911 \\
\hline Camera & 118.7228 & 118.6388 & 118.4387 & 62.3434 & 62.6854 & 62.5541 \\
\hline
\end{tabular}

Lena Goldhill Cameraman Mandrill
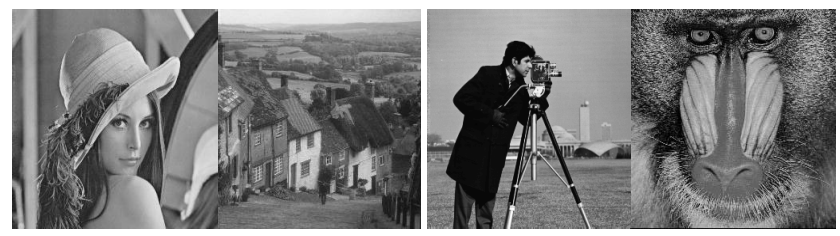

(a) Original cover images

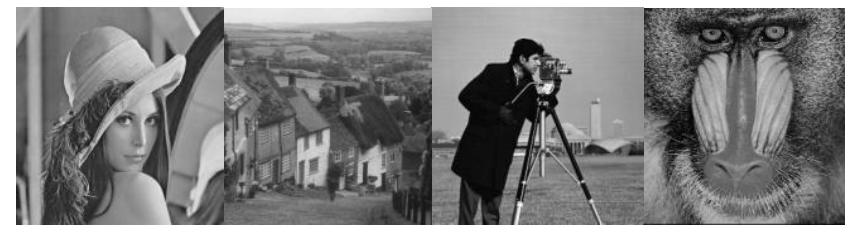

(b) Stego images obtained with the proposed method in [16]

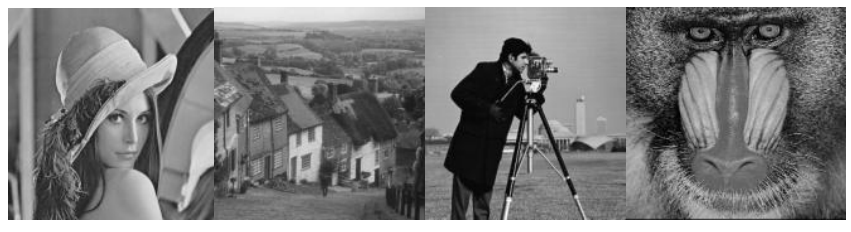

(c) Stego images obtained with the proposed method

Fig 3: Results of Experimental Validation

\section{CONCLUSION}

In this study, an attempt is made to develop a new approach for data hiding in grey scale images in which data encryption, compression and hiding are combined to enhance bandwidth utilization and achieve secure communication. First layer of security is achieved by using a symmetric key based on the concept of a flexible matrix. The data hiding capacity of the cover image is enhanced by encrypting as well as compressing the ciphered data using CRT. In this algorithm, upper $5^{\text {th }} \& 6^{\text {th }}$ bits of the image pixel are also used for post pixel adjustment to further improve the visual perception of the stego image. In fact, by doing so, an additional layer of security to the original message is also provided besides increasing data hiding capacity of the cover image. The proposed algorithm requires less computational overhead as compared to other steganographic techniques which transform the cover image into frequency domain. Experiment results have demonstrated that the proposed scheme for data hiding works satisfactorily for different gray level digital images. Moreover, the proposed method provides acceptable image quality with very little distortion in the cover image.
Lena Goldhill Cameraman Mandrill

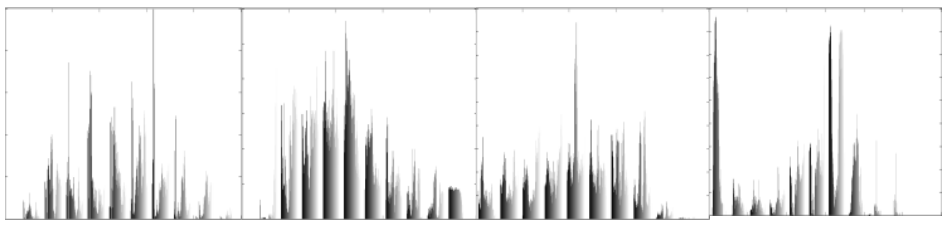

(a) Histograms of original cover images

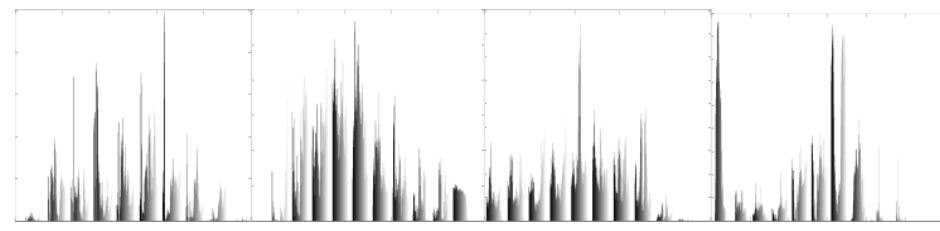

(b) Histograms of stego images obtained with the proposed method [16]

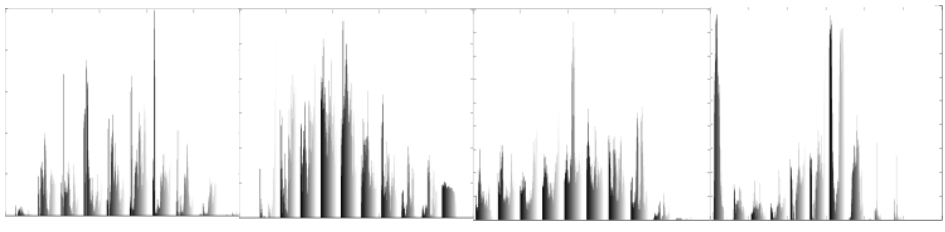

(c) Histograms of stego images obtained with the proposed method

Fig 4: Histograms of cover \& stego-images

\section{REFERENCES}

[1] Forouzan. B. A., Cryptography and Network Security. $4^{\text {th }}$ ed. Publisher: McGraw-Hill Higher Education, 2008.

[2] Vinoly Seromony, "Image encryption and compression using number theoretic paradigm", GSPx Conference, (April 2003).

[3] W.B. Pennebaker, J. Mitchell, "JPEG still image compression standard", New York, Van Nostrand Reinhold, edition (2001).

[4] V. Jagannathan, A. Mahadevan, R. Hariharan and E. Srinivasan, "Number Theory Based Image Compression Encryption and Application to Image Multiplexing", IEEE - ICSCN 2007, MIT Campus, Anna University, Chennai, India. Feb. 22-24, (2007), pp.59-64.

[5] Neil F. Johnson and Sushil Jajodia, "Exploring steganography seeing the unseen", IEEE Computer, (February 1998), pp. 26-34.

[6] R.J. Anderson and F. A. P. Petitcolas, "On the limits of the steganography", IEEE Journal Selected Areas in Communications, (2001), 16(4), pp. 474-481. 
[7] F. A. P. Petitcolas, R.J. Anderson and M. G. Kuhn, "Information Hiding-A Survey", Proceedings of IEEE, (July 1999), vol. 87 pp. 1062-1078.

[8] K. B. Raja, C. R. Chowdary, R. K. Venugopal, L. M Patnaik, "A Secure Image Steganography using LSB, DCT and Compression Techniques on Raw Images", Proceedings of IEEE, (2005), pp. 171-176.

[9] Westfeld A., "Steganography for Radio Amateurs-A DSSS Based Approach for Slow Scan Television", LNCS 4437, Springer-Verlag Berlin Heidelberg, 2007, pp. 201-215.

[10] Y. K. Lee and L. H. Chen, "A high capacity image steganographic model", In IEE Vision, Image and Signal Processing, (2000), 147(3), pp. 288-294.

[11] Michael Backes, "A cryptographically sound Dolev Yao style security proof of the Otway-Rees protocol", In Proceedings of the 9th European Symposium on Research in Computer Security (ESORICS), vol. 3193, Springer Verlag, Berlin Germany, (September 2004), pp. 89-108.

[12] L. M. Marvel, C. G. Boncelet, and C. T. Retter, "Spread spectrum image steganography", IEEE Transactions on Image Processing, vol. 8, Aug. (1999), pp. 1075-1083.
[13] Xin Li, Hong-heather Yu, "Transparent and Robust Audio Data Hiding in Spectrum Domain", IEEE International Conference on Multimedia and Expo (ICME), NY, USA, (2 August 2000).

[14] Chin-Chen Chang, Chi-Shiang Chan,Yi-Hsuan Fan, "Image hiding scheme with modulus function and dynamic programming strategy on partitioned pixels," Pattern Recognition, vol. 39, (2006), pp. 1155-1167.

[15] Balkrishan and Amar Partap Singh, "Hiding Encrypted Data using Randomly Chosen Moderate Bit Insertion in Digital Image Steganography", Journal of Computer Science and Engineering, vol. 1, issue 2, (June 2010), pp. 21-27.

[16] Jindal B, Singh AP (2013) Camouflaging in Digital Image for Secure Communication. J. IE(I)-Springer: Electrical, Electronics \& Telecommunication and Computer Engineering, vol. 94, no. 2, pp 85-92, June 2013.

[17] Gonzalez R C and Woods R E. Digital Image Processing. Pearson Education, (2003). 